\title{
RESOLVED SPECTROSCOPY OF A BROWN DWARF BINARY AT THE T DWARF/Y DWARF TRANSITION*
}

\author{
Adam J. Burgasser ${ }^{1}$, Christopher R. Gelino ${ }^{2}$, Michael C. Cushing ${ }^{3}$, And J. Davy Kirkpatrick ${ }^{2}$ \\ ${ }^{1}$ Center for Astrophysics and Space Science, University of California San Diego, La Jolla, CA 92093, USA; aburgasser@ucsd.edu \\ ${ }^{2}$ Infrared Processing and Analysis Center, MC 100-22, California Institute of Technology, Pasadena, CA 91125, USA \\ ${ }^{3}$ Ritter Astrophysical Research Center, University of Toledo, MS 113, Toledo, OH 43606, USA \\ Received 2011 August 27; accepted 2011 October 18; published 2011 December 28
}

\begin{abstract}
We report resolved near-infrared imaging and spectroscopic observations of the T8.5 binary WISEP J045853.90+643452.6AB obtained with Keck/NIRC2, Keck/OSIRIS, and the Keck Laser Guide Star Adaptive Optics system. These data confirm common proper and radial motion for the two components, and we see the first indications of orbital motion (mostly radial) for this system. $H$-band spectroscopy identifies both components as very late type brown dwarfs with strong $\mathrm{H}_{2} \mathrm{O}$ and $\mathrm{CH}_{4}$ absorption. The spectrum of WISE J0458+6434B also exhibits a compelling signature of $\mathrm{NH}_{3}$ absorption over 1.52-1.54 $\mu \mathrm{m}$ when compared to the T9 dwarf UGPS J072227.51-054031.2. Comparison to T8-Y0 spectral standards and $H$-band spectral indices indicate classifications of T8.5 and T9.5 for these two components, approaching the boundary between the T dwarf and Y dwarf spectral classes.
\end{abstract}

Key words: binaries: visual - brown dwarfs - stars: individual (WISEP J045853.90+643452.6) - stars: low mass

Online-only material: color figures

\section{INTRODUCTION}

The lowest-temperature brown dwarfs-the L, T, and Y dwarfs - span an astrophysically rich parameter space of mass, temperature, and atmospheric chemistry that bridges the lowestmass stars and the warmest exoplanets (Kirkpatrick 2005 and references therein). These sources also probe the efficiency of low-mass star formation and the chemical enrichment history of the Galaxy (Burgasser 2004; Metchev et al. 2008; Bate 2009). Very low temperature brown dwarfs down to effective temperatures $\left(T_{\text {eff }} \mathrm{s}\right)$ of $\sim 300-500 \mathrm{~K}$ are now being identified (e.g., Lucas et al. 2010; Luhman et al. 2011; Liu et al. 2011; Cushing et al. 2011; Kirkpatrick et al. 2011), primarily in deep, wide-field infrared surveys such as the United Kingdom Infrared Telescope Deep Sky Survey (UKIDSS; Lawrence et al. 2007); the Canada-France Brown Dwarf Survey (CFBDS; Delorme et al. 2008b); and, most recently, the Wide-field Infrared Survey Explorer (WISE; Wright et al. 2010). These discoveries probe previously unexplored realms of gas and condensate atmospheric chemistry (Burrows et al. 2003; Lodders \& Fegley 2006), and encompass the newly identified Y dwarf spectral class (Cushing et al. 2011).

While changes in spectral morphology are largely tied to changes in temperature, the transitions between late-type spectral classes also trace significant changes in atmospheric chemistry and dynamics. The $\mathrm{M}$ dwarf to $\mathrm{L}$ dwarf transition is accompanied by the formation of mineral condensates (Tsuji et al. 1996; Cooper et al. 2003; Helling et al. 2008), which deplete the photosphere of metal oxide gases ( $\mathrm{TiO}, \mathrm{VO}$ ) and lead to more reddened optical and near-infrared spectral energy distributions. In addition, magnetic coronal and chromospheric emission drops precipitously across this $\mathrm{M} / \mathrm{L}$ transition (Gizis et al. 2000; West et al. 2004), the result of both increasingly

\footnotetext{
* Data presented herein were obtained at the W. M. Keck Observatory, which is operated as a scientific partnership among the California Institute of Technology, the University of California, and the National Aeronautics and Space Administration, and made possible by the generous financial support of the W. M. Keck Foundation.
}

neutral atmospheres (Gelino et al. 2002; Mohanty et al. 2002) and reduced magnetic field energy with reduced mass (Christensen et al. 2009). The L dwarf to T dwarf transition, signaled by the emergence of $\mathrm{CH}_{4}$ absorption at near-infrared wavelengths, is coincident with the removal of mineral condensate clouds from the photosphere (Ackerman \& Marley 2001; Burgasser et al. 2002b; Knapp et al. 2004; Tsuji 2005) and the increased influence of non-equilibrium chemistry on observed molecular abundances (Noll et al. 1997; Griffith \& Yelle 1999; Saumon et al. 2006; Yamamura et al. 2010). Given the very recent discovery of $Y$ dwarfs, transitional behaviors have yet to be explored at these spectral types, although atmospheric models predict the formation of salt and water condensates that may significantly shift photospheric chemistry and spectral appearance (e.g., Lodders 1999; Burrows et al. 2003; Leggett et al. 2007).

Ideal probes of such spectral transitions are physical binaries whose components straddle the corresponding classes. These presumably coeval systems share a common age and bulk chemical composition, and reside at a common distance, making relative comparisons less dependent on these parameters. For example, evidence for a rapid dissipation of clouds at the L dwarf/T dwarf transition includes the apparent brightening of early $\mathrm{T}$ dwarfs at $1 \mu \mathrm{m}$ as observed among $\mathrm{L} / \mathrm{T}$ transition binaries (Burgasser et al. 2006b; Liu et al. 2006; Looper et al. 2008), and the higher apparent multiplicity rate of these systems (Burgasser 2007). Physical parameters such as age, mass, and radii have also been measured for low-mass binaries; these serve as critical empirical constraints for evolutionary models (e.g., Lane et al. 2001; Zapatero Osorio et al. 2004; Stassun et al. 2006; Dupuy et al. 2009; Konopacky et al. 2010). Finally, companions to low-luminosity sources are frequently low-temperature extrema. The prototypes for both the L dwarf (GD 165B; Becklin \& Zuckerman 1988) and T dwarf (Gliese 229B; Nakajima et al. 1995) spectral classes were identified as companions to low-luminosity stars, as were two of the coldest brown dwarfs currently known, WD 0806-661B (Luhman et al. 2011) and CFBDSIR J1458+1013B (Liu et al. 2011). 
One of the first very late type T dwarfs to be uncovered by the WISE survey was WISEP J045853.90+643452.6 (hereafter WISE J0458+6434; Mainzer et al. 2011), a T8.5 dwarf at an estimated distance of $10 \mathrm{pc}$ from the Sun. Subsequent adaptive optics (AO) imaging observations by Gelino et al. (2011) revealed this source to be a binary with a separation of 0.5 and a relative brightness of $\Delta J=0.98 \pm 0.08$. Given the late spectral type of the composite spectrum and the significant magnitude difference between the components, WISE J0458+6434B was estimated to have a T9 spectral type and $T_{\text {eff }} \approx 500 \mathrm{~K}$. However, uncertainties in the distance of this system, the unknown spectral energy distribution of the secondary, and the very recent empirical definition of the $\mathrm{Y}$ dwarf spectral class (Cushing et al. 2011) mean that WISE J0458+6434AB could potentially straddle the T dwarf/Y dwarf transition.

In this article, we present resolved near-infrared imaging and spectroscopy of the WISE J0458+6434 system obtained with the Keck II NIRC2 camera, OH-Suppressing InfraRed Integral field Spectrograph (OSIRIS; Larkin et al. 2006), and Laser Guide Star Adaptive Optics system (LGSAO; Wizinowich et al. 2006; van Dam et al. 2006). These data confirm common proper motion for the system and the first evidence for orbital motion. We use the $H$-band OSIRIS spectra to classify the components and identify tentative evidence for near-infrared $\mathrm{NH}_{3}$ absorption in WISE J0458+6434B. In Section 2 we describe our observations and data reduction methods. In Section 3 we analyze our astrometric measurements and provide an initial qualitative characterization of the binary orbit. In Section 4 we analyze the spectral data, deriving classifications using both spectral templates and $H$-band indices, and examining evidence for $\mathrm{NH}_{3}$ absorption. In Section 5 we discuss our results, focusing on the absolute magnitudes of WISE J0458+6434AB in the context of the T dwarf/Y dwarf transition.

\section{OBSERVATIONS}

\subsection{NIRC2 Imaging}

New high-resolution LGSAO imaging observations of WISE J0458+6434 were obtained with NIRC 2 on the $10 \mathrm{~m} \mathrm{Keck}$ II Telescope on 2011 August 29 (UT). Conditions were clear with seeing of 0.5 . Data were obtained with the narrow plate scale mode of NIRC2 (9.963 \pm 0.011 mas pixel $^{-1}$; Pravdo et al. 2006) at an orientation of $0^{\circ}$ and air mass $\approx 1.5$. We observed the target in the $J$-, $H$ - and $K_{s}$-band filters using a three-point dither pattern that avoided the noisy, lower left quadrant of the focal plane array. Individual exposure times were $120 \mathrm{~s}$ in $J$ and $K_{s}$ and $300 \mathrm{~s}$ in $H$, for total integrations of $360 \mathrm{~s}, 900 \mathrm{~s}$, and $360 \mathrm{~s}$ in $J, H$, and $K_{s}$, respectively. The sodium LGS provided the wave front reference source for AO correction, while tip-tilt aberrations and quasi-static changes were measured by monitoring the $R=15.6$ field star USNO-B1.0 1545-0122611 (Monet et al. 2003) located $\rho=27^{\prime \prime} .7$ from WISE J0458+6434.

Images were reduced using custom IDL ${ }^{4}$ scripts. Sky background and dark current were removed from each image by pairwise subtracting it with the following image in the dither sequence, then dividing by a normalized dome flat to correct for pixel-to-pixel sensitivity variations. The calibrated image frames were registered to the peak of the primary's point-spread function (PSF), then median-combined to produce the final mosaics shown in Figure 1.

\footnotetext{
4 Interactive Data Language.
}

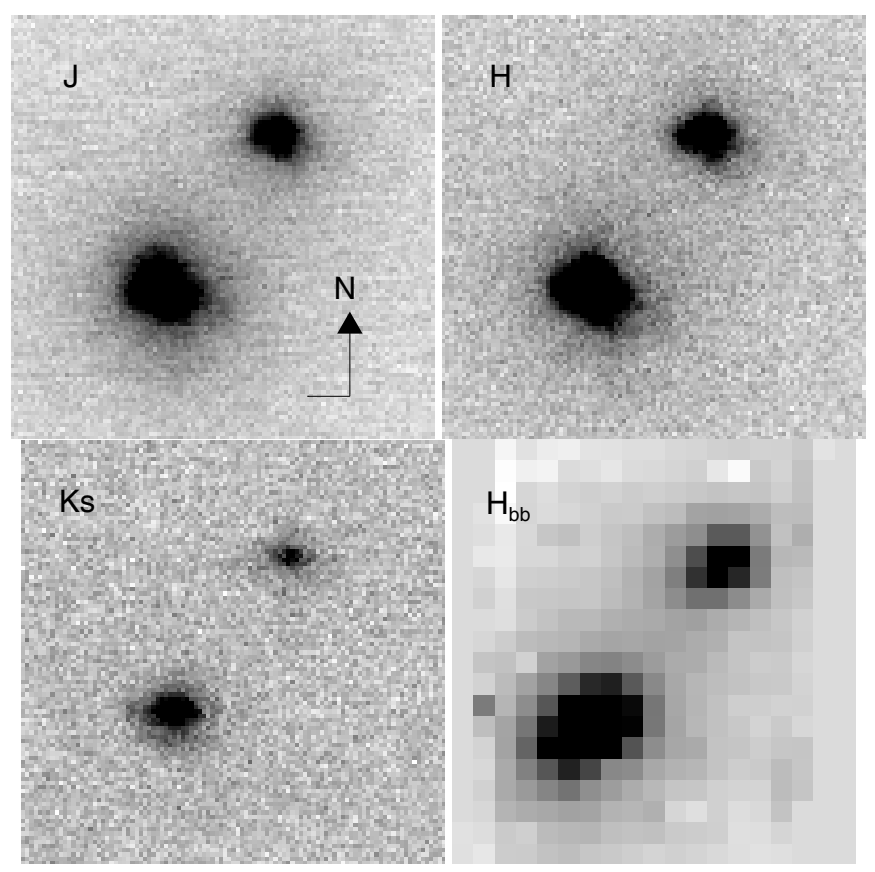

Figure 1. Images of the WISE J0458+6434AB system, from NIRC2 $J$ - (top left), $H$ - (top right), and $K_{s}$-band (bottom left) observations obtained on 2011 August 29 (UT), and OSIRIS observations (bottom right) obtained on 2011 February 3 (UT). The last image was generated by combining the OSIRIS data cube over wavelengths $1.56-1.60 \mu \mathrm{m}$. All fields are $1^{\prime \prime}$ on a side and oriented with north up and east to the left.

The quality of these data is significantly improved over the discovery observations from Gelino et al. (2011), with components that are well separated in the field of view (FOV). Relative fluxes were computed using aperture photometry on the coadded images. Given the wide separation of the components, no PSF subtraction or other specialized procedures were required for the photometry. The radial separation and position angle of the fainter component with respect to the brighter component was measured on each of the nine individual exposures. The values listed in Table 1 report the average and standard deviation of those nine measurements. Relative $J$ - and $H$-band flux measurements are in excellent agreement with those reported in Gelino et al. (2011), but are of far greater precision. In contrast, there is a significant shift in the relative separation of the two components, with $\Delta \alpha=54 \pm 14$ mas and $\Delta \delta=27 \pm 16$ mas. Notably, this shift is largely in a radial direction. We discuss the astrometry in further detail in Section 3.

\subsection{OSIRIS Spectroscopy}

Resolved spectroscopy of WISE J0458+6434 was obtained with OSIRIS and LGSAO on 2011 February 3 (UT), in clear conditions with seeing of $0 .{ }^{\prime} 8-1^{\prime \prime} .0$. The 50 mas-scale camera and $\mathrm{H}_{b b}$ filter were employed, providing $1.47-1.80 \mu \mathrm{m}$ spectroscopy at an average resolution of 3800 and dispersion of $2.1 \AA$ pixel $^{-1}$ over a 0 ". $8 \times 3$ ". 2 FOV. The source was first acquired in the imaging camera, then offset to the spectrograph array. The image rotator was set to $0^{\circ}$. Eight exposures of $600 \mathrm{~s}$ each were obtained over an air-mass range of 1.40-1.52 using a two-step dither pattern with steps of 1".0 along the long axis of the FOV. For LGSAO tip-tilt correction, we employed the same star as that used for the NIRC2 imaging. We also obtained four $30 \mathrm{~s}$ observations of the A0 V star BD +64 $489(V=9.43)$ in natural guide star AO mode at an air mass of 1.72 for telluric correction and flux calibration purposes. 
Table 1

Astrometry for WISE J0458+6434AB

\begin{tabular}{|c|c|}
\hline Parameter & Value \\
\hline \multicolumn{2}{|c|}{ NIRC2 Epoch 2010 Mar $24\left(\right.$ UT) ${ }^{\mathrm{a}}$} \\
\hline$\Delta \alpha \cos \delta($ mas $)$ & $-328 \pm 14$ \\
\hline$\Delta \delta$ (mas) & $390 \pm 16$ \\
\hline$\rho$ (mas) & $510 \pm 20$ \\
\hline$\theta(\operatorname{deg})$ & $320 \pm 1$ \\
\hline$\Delta J$ & $0.98 \pm 0.08$ \\
\hline$\Delta H$ & $1.00 \pm 0.09$ \\
\hline \multicolumn{2}{|c|}{ OSIRIS Epoch 2011 Feb 3 (UT) } \\
\hline$\Delta \alpha \cos \delta(\mathrm{mas})$ & $-308 \pm 10$ \\
\hline$\Delta \delta$ (mas) & $386 \pm 14$ \\
\hline$\rho$ (mas) & $493 \pm 15$ \\
\hline$\theta(\mathrm{deg})$ & $321.4 \pm 1.0$ \\
\hline \multicolumn{2}{|c|}{ NIRC2 Epoch 2011 Aug 29 (UT) } \\
\hline$\Delta \alpha \cos \delta(\mathrm{mas})$ & $-274.5 \pm 3.4$ \\
\hline$\Delta \delta$ (mas) & $362.9 \pm 4.0$ \\
\hline$\rho$ (mas) & $455.1 \pm 4.2$ \\
\hline$\theta(\operatorname{deg})$ & $322.9 \pm 0.4$ \\
\hline$\Delta J$ & $0.98 \pm 0.01$ \\
\hline$\Delta H$ & $1.02 \pm 0.01$ \\
\hline$\Delta K_{s}$ & $1.06 \pm 0.03$ \\
\hline
\end{tabular}

Notes. Angular separation $(\rho)$ and position angle $(\theta)$ are measured from the brighter primary to the fainter secondary. ${ }^{a}$ From Gelino et al. (2011).

Data were reduced with the OSIRIS data reduction pipeline (Krabbe et al. 2004), version 2.3. We first subtracted from the source and calibrator images median-combined sets of dark frames with identical exposure times. We then used the pipeline to adjust bias levels, remove detector artifacts and cosmic rays, extract and wavelength-calibrate the position-dependent spectra (using the most current rectification files as of 2011 February), assemble three-dimensional data cubes, and correct for dispersion. Figure 1 displays a $1^{\prime \prime} \times 1^{\prime \prime}$ section of this cube co-added over the wavelength range 1.56-1.60 $\mu \mathrm{m}$. The two components are cleanly resolved, separated by $\sim 10$ spaxels $(0,5)$ along a NW-SE axis. The full width at half-maximum of the PSF was measured to be 150 mas (3 spaxels) over this wavelength range for both components, slightly elongated in the direction of the tip-tilt star. Relative astrometry from these data were determined from each of the reduced data cubes, using a median stack over the spectral dimension 1.56-1.60 $\mu \mathrm{m}$. The IDL routine $\mathrm{cntrd}$ was used to measure the peak pixel positions of the primary and secondary in each image, and pixel separations along each axis were converted to angular separations in right ascension and declination. Mean measurements and standard deviations are listed in Table 1. The offsets are intermediate between those of the two NIRC2 observations, consistent with a continuous reduction in separation over nearly constant position angle.

Spectra for both components of WISE J0458+6434 and the calibrator star were extracted directly from the data cube via aperture photometry in each image plane, using a 1.5 spaxel (75 mas) aperture for WISE J0458+6434 and a 3 spaxel (150 mas) aperture for BD +64 489, with 10-20 spaxel ( $\left(0.5-1{ }^{\prime \prime} 0\right)$ sky annuli. The individual spectra for all three sources were scaled and combined using the xcombspec routine in SpeXtool (Cushing et al. 2004). Flux calibration and telluric correction of the WISE J0458+6434AB spectra were performed using the xtellcor_general routine in SpeXtool, assuming a $20 \mathrm{~nm}$ Gaussian kernel to model the widths of the A0V H I lines,

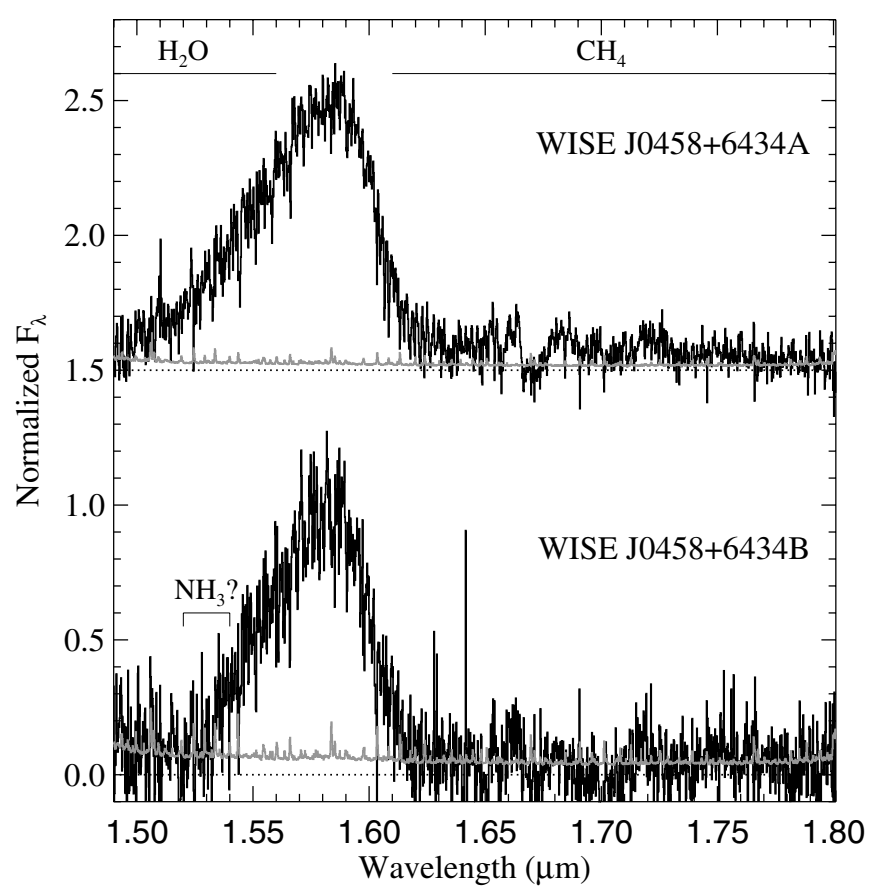

Figure 2. Reduced OSIRIS spectra (black lines) of WISE J0458+6434A (top) and B (bottom) over 1.5-1.8 $\mu \mathrm{m}$. Spectra are normalized at $1.58 \mu \mathrm{m}$, with data for WISE J0458+6434A offset by 1.5 units for clarity (dotted lines). Uncertainty spectra are indicated by gray lines. Regions of strong $\mathrm{H}_{2} \mathrm{O}$ and $\mathrm{CH}_{4}$ absorption bands are labeled, as well as the 1.52-1.54 $\mu \mathrm{m}$ region of $\mathrm{NH}_{3}$ absorption tentatively indicated in the spectrum of WISE J0458+6434B.

and a Kurucz model spectrum of Vega (Vacca et al. 2003). Figure 2 displays the reduced spectra of the WISE J0458+6434 components. Both are unambiguously late-type $\mathrm{T}$ dwarfs based on the presence of strong $\mathrm{H}_{2} \mathrm{O}$ absorption up to $1.55 \mu \mathrm{m}$ and strong $\mathrm{CH}_{4}$ absorption beyond $1.59 \mu \mathrm{m}$. These bands constrain the $H$-band flux to a discrete $1.50-1.62 \mu \mathrm{m}$ peak, which is narrower in the spectrum of WISE J0458+6434B. Unbinned peak signal-to-noise ratio $(\mathrm{S} / \mathrm{N})$ is $\sim 40$ in the spectrum of WISE J0458+6434A and $\sim 15$ in the spectrum of WISE J0458+6434B.

\section{ASTROMETRIC ANALYSIS}

As noted above, astrometry from NIRC2 and OSIRIS observations of WISE J0458+6434 indicate a monotonic decrease in the separation of this pair along a near constant position angle of $320^{\circ}$, with the separations in right ascension from the two NIRC 2 observations differing by nearby $4 \sigma$. To assess how this relative motion relates to systemic common motion, and hence physical association, we compared our measurements against predicted separations anchored to our 2011 August 29 (UT) astrometry, considering three limiting scenarios: (1) the secondary is a background source with negligible parallactic or proper motion, (2) the secondary is at the same distance as the primary, but with negligible proper motion, and (3) the primary and secondary maintain constant separation at all times. Figure 3 shows that none of these scenarios are fully consistent with the data, although the last provides the closest match. Allowing relative motions and distances to vary freely, we found that our measured astrometry (with $3 \sigma$ uncertainty allowance) constrains the

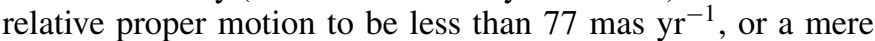
$4 \mathrm{~km} \mathrm{~s}^{-1}$ at a distance of $10 \mathrm{pc}$. For comparison, the circular orbital speed of a $0.05 M_{\odot}$ brown dwarf in an equal-mass binary separated by $5 \mathrm{AU}$ is $\sim 3 \mathrm{~km} \mathrm{~s}^{-1}$. We also measured the 

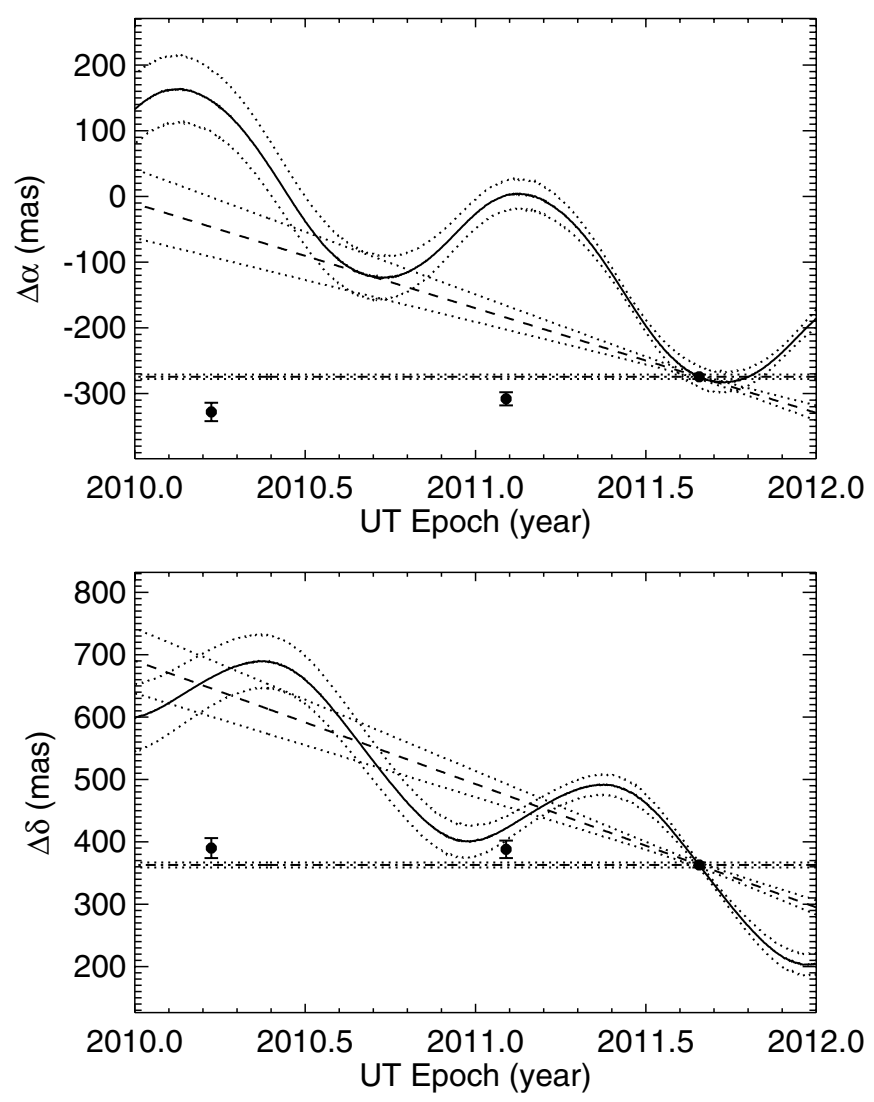

Figure 3. Separation of the WISE J0458+6434 components in right ascension (top) and declination (bottom) as a function of time, as measured from primary to secondary. Measurements from NIRC 2 and OSIRIS are indicated by points with error bars. Lines delineate predicted separations relative to epoch 2011 August 29 (UT) astrometry assuming that (1) the secondary is a distant background source (solid line), (2) the secondary is an unmoving source at the same distance as the primary (dashed line), and (3) the separation between primary and secondary remains constant. Dotted lines indicate $1 \sigma$ uncertainties in these trajectories based on uncertainties in the system's distance, total proper motion, and separation at epoch 2011 August 29 (UT).

relative radial velocity $(\Delta \mathrm{RV})$ of the two components by crosscorrelating our OSIRIS spectra over the 1.58-1.65 $\mu \mathrm{m}$ range, properly accounting for noise through Monte Carlo simulation. We find $\Delta \mathrm{RV}=3 \pm 15 \mathrm{~km} \mathrm{~s}^{-1}$, consistent with zero to within $1 / 5$ of a spectral resolution element.

These stringent limits on the relative motion of the WISE J0458+6434 components indicate that the two sources comprise a common proper motion, physically bound binary. As such, the small but significant astrometric shifts observed must arise from orbital motion. Figure 4 provides a visualization of our three separation measurements projected onto the plane of the sky. The near radial motion and marginal indication of an inward acceleration $\left(\ddot{\rho}=-1.6 \pm 1.3 \mathrm{~km} \mathrm{~s}^{-1} \mathrm{yr}^{-1}\right)$ suggests an eccentric and/or nearly edge-on orbit that is moving toward alignment. In the most optimistic case-if WISE J0458+6434 was observed at or near maximum elongation in the first NIRC2 epoch-these observations constrain the orbital semimajor axis to the range $5 /(1+e) \lesssim a \lesssim 5 /(1-e) \mathrm{AU}$, where $e$ is the orbital eccentricity and a distance of $10 \mathrm{pc}$ is assumed. Unfortunately, more stringent constraints on this system's orbit are not possible with these few measurements clumped in one region of separation space. Over the next decade it should be possible to disentangle the eccentricity and inclination of the orbit, and provide more robust constraints on the period and total system mass.

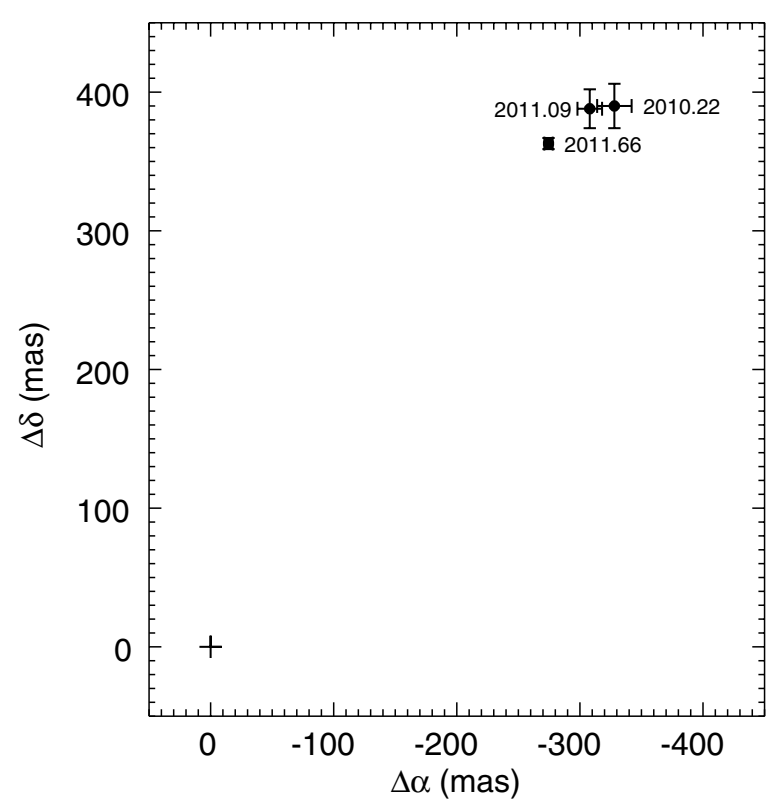

Figure 4. Relative separations of WISE J0458+6434A (cross) and B (data points) projected onto the plane of the sky. The trend in motion is nearly radial, toward a smaller separation.

\section{SPECTRAL ANALYSIS}

\subsection{Component Spectral Classifications}

The strong $\mathrm{H}_{2} \mathrm{O}$ and $\mathrm{CH}_{4}$ absorption in the spectra of WISE J0458+6434AB are consistent with very late T and possibly Y spectral types. To robustly determine the classifications, we first compared the spectra to the standards 2MASS J04151954-0935066 (T8; hereafter 2MASS J0415-0935; Burgasser et al. 2002a, 2004), UGPS J072227.51-054031.2 (T9; hereafter UGPS J0722-0540; Lucas et al. 2010; Cushing et al. 2011), and WISEP J173835.52+273258.9 (Y0; hereafter WISE J1738+2732; Cushing et al. 2011). The latter two sources were defined by Cushing et al. (2011) to extend the T dwarf nearinfrared classification scheme of Burgasser et al. (2006a) across the T dwarf/Y dwarf transition. Figure 5 shows these comparisons, with all of the spectra smoothed to a common resolution of $\lambda / \Delta \lambda=100$ and normalized at $1.58 \mu \mathrm{m}$. WISE J0458+6434A is a reasonably fair match to 2 MASS J0415-0935, albeit with slightly deeper absorption on the blue side of the $1.58 \mu \mathrm{m}$ peak and a somewhat narrower peak. It has weaker $1.6 \mu \mathrm{m}$ $\mathrm{CH}_{4}$ absorption than UGPS J0722-0540, indicating that it is not as late as T9. WISE J0458+6434B, on the other hand, has stronger $\mathrm{H}_{2} \mathrm{O}$ and $\mathrm{CH}_{4}$ absorption than 2MASS J0415-0935 and UGPS J0722-0540, but less absorption around $1.55 \mu \mathrm{m}$ than WISE J1738+2732. These by-eye comparisons suggest types of T8-T8.5 for WISE J0458+6434A and T9-T9.5 for WISE J0458+6434B.

We also computed $H$-band spectral classification indices $\mathrm{H}_{2} \mathrm{O}-\mathrm{H}$ and $\mathrm{CH}_{4}-\mathrm{H}$ (Burgasser et al. 2006a) and $\mathrm{NH}_{3}-\mathrm{H}$ (Delorme et al. 2008a) for both components of WISE J0458+6434. The last index was shown by Cushing et al. (2011) to exhibit a distinct break at the $\mathrm{T} / \mathrm{Y}$ transition. Values are listed in Table 2, along with estimated subtypes based on comparison to index values for the spectral standards. Indices for WISE J0458+6434A are well matched to those for UGPS J0722-0540, albeit with an $\mathrm{NH}_{3}-\mathrm{H}$ index intermediate between $\mathrm{T} 8$ and $\mathrm{T} 9 . \mathrm{H}_{2} \mathrm{O}-\mathrm{H}$ and $\mathrm{NH}_{3}-\mathrm{H}$ indices for WISE J0458+6434B are intermediate between T9 and Y0; 

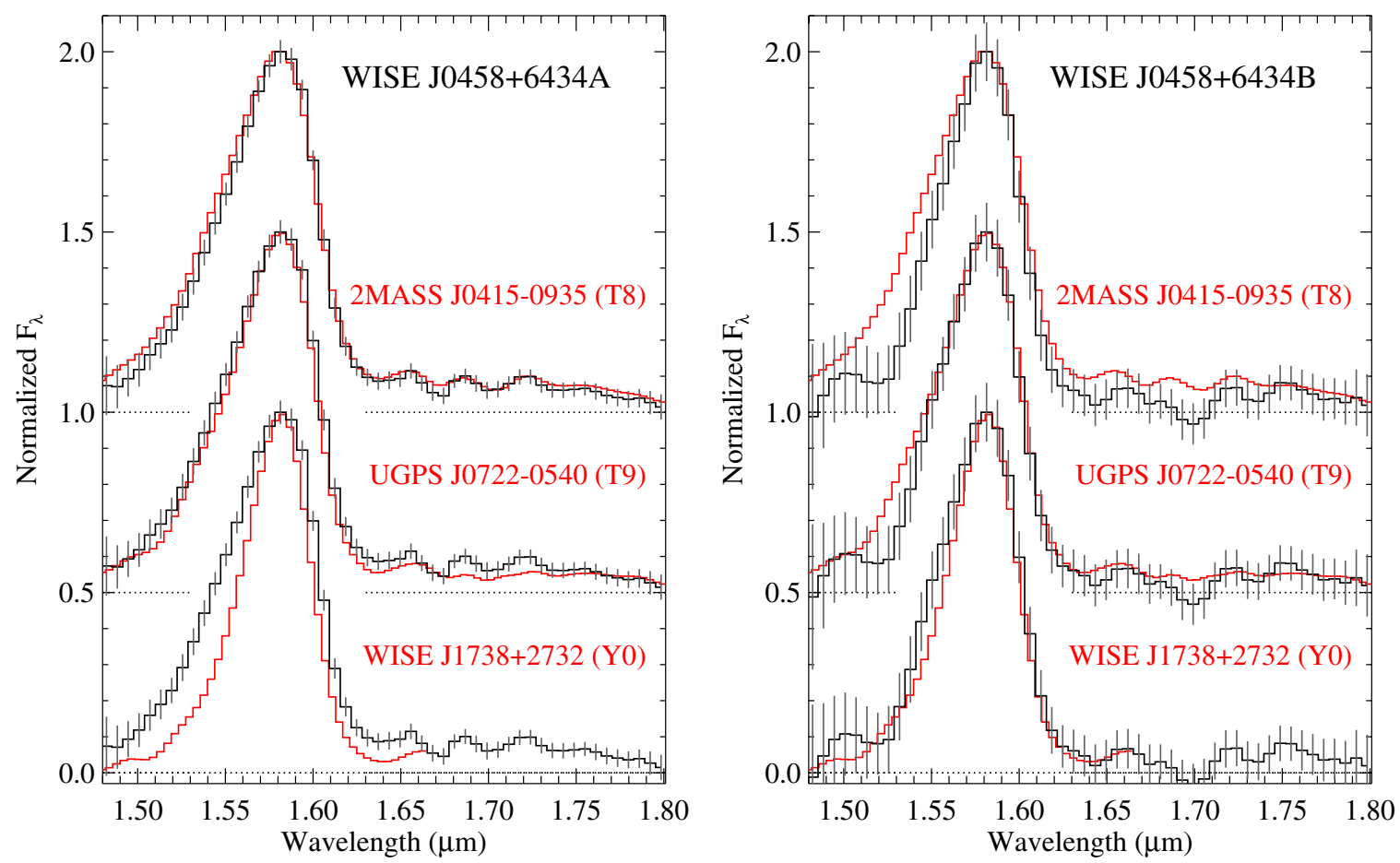

Figure 5. Boxcar-smoothed OSIRIS spectra (black lines) of WISE J0458+6434A (left) and WISE J0458+6434B (right) compared to spectral standards (red lines) 2MASS J0415-0935 (T8, top), UGPS J0722-0540 (T9, middle), and WISE J1738+2732 (Y0, bottom). All spectra are smoothed to a common resolution of $\lambda / \Delta \lambda=100$ and normalized at $1.58 \mu \mathrm{m}$. Uncertainties in the smoothed WISE J0458+6434AB spectra are indicated by error bars; note that these do not take into account correlated errors. Zero points are indicated by dotted lines.

(A color version of this figure is available in the online journal.)

Table 2

Spectral Indices and Classification

\begin{tabular}{|c|c|c|c|c|c|}
\hline Source & $\mathrm{H}_{2} \mathrm{O}-\mathrm{H}^{\mathrm{a}}$ & $\mathrm{CH}_{4}-\mathrm{H}^{\mathrm{a}}$ & $\mathrm{NH}_{3}-\mathrm{H}^{\mathrm{a}}$ & $\begin{array}{l}\text { Adopted } \\
\text { SpT }^{\mathrm{b}}\end{array}$ & Ref. \\
\hline 2 MASS J0415-0935 & $0.183 \pm 0.002$ & $0.104 \pm 0.002$ & $0.628 \pm 0.004$ & $\mathrm{~T} 8$ & 1,2 \\
\hline WISE J0458+6434A & $0.129 \pm 0.006(\mathrm{~T} 9)$ & $0.079 \pm 0.003(\mathrm{~T} 9)$ & $0.561 \pm 0.003(\mathrm{~T} 8.5)$ & T8.5 & 3 \\
\hline UGPS J0722-0540 & $0.115 \pm 0.006$ & $0.075 \pm 0.005$ & $0.527 \pm 0.008$ & T9 & 4,5 \\
\hline WISE J0458+6434B & $0.086 \pm 0.015(\mathrm{~T} 9.5)$ & $0.040 \pm 0.007(\mathrm{Y} 0+)$ & $0.459 \pm 0.008(\mathrm{~T} 9.5)$ & T9.5 & 3 \\
\hline WISE J1738+2732c & $0.045 \pm 0.008$ & $0.052 \pm 0.008$ & $0.350 \pm 0.011$ & Y0 & 5 \\
\hline
\end{tabular}

Notes.

${ }^{a}$ Index uncertainties computed directly from spectral flux errors via Monte Carlo sampling.

${ }^{\mathrm{b}}$ For WISE J0458+6434AB, this includes comparison to spectral standards as shown in Figure 5.

${ }^{\mathrm{c}}$ Spectroscopic standards (Burgasser et al. 2006a; Cushing et al. 2011).

References. (1) Burgasser et al. 2002a; (2) Burgasser et al. 2006a; (3) This paper; (4) Lucas et al. 2010; (5) Cushing et al. 2011.

its $\mathrm{CH}_{4}-\mathrm{H}$ index is smaller than any late $\mathrm{T}$ dwarf/Y dwarf measured thus far with the exception of the T9.5 WISEPC J014807.25-720258.8 (Cushing et al. 2011). Combining the index types and direct spectral comparisons, we infer subtypes of T8.5 and T9.5 for WISE J0458+6434A and B.

\subsection{Ammonia in the Spectrum of WISE J0458+6434B}

Close examination of the spectral comparisons in Figure 5 indicates additional absorption in the spectrum of WISE J0458+6434B over the 1.52-1.54 $\mu \mathrm{m}$ region compared to both 2MASS J0415-0935 and UGPS J0722-0540. This feature is similar to those noted by Cushing et al. (2011) in the spectra of the Y0 dwarfs WISEPC J140518.40+553421.5 and WISE J1738+2732, which were tentatively attributed to $\mathrm{NH}_{3}$ absorption (see also Bochanski et al. 2011). We examined whether the excess absorption in WISE J0458+6434B could also be due to $\mathrm{NH}_{3}$ by comparing its spectrum and that of UGPS J0722-0540 to absorption coefficients $(\alpha[\lambda])$ for $\mathrm{H}_{2} \mathrm{O}, \mathrm{CH}_{4}$, and $\mathrm{NH}_{3}$. Absorption coefficients were computed by multiplying scattering cross-sections per molecule $(\sigma[\lambda])$, as tabulated by Freedman et al. (2008) for $T=500 \mathrm{~K}$ and $P=1 \mathrm{bar}$, with molecular number densities $\left(n_{i}\right)$ assuming fractional abundances $\left(f_{i}\right)$ based on the nonequilibrium chemistry calculations of Saumon et al. (2006):

$$
\begin{aligned}
\alpha_{i}[\lambda] & =\sigma_{i}[\lambda] n_{i} \\
& =\sigma_{i}[\lambda] f_{i} n .
\end{aligned}
$$

Here, $n=1.4 \times 10^{19} \mathrm{~cm}^{-3}$ is the total number density of gas particles assuming an ideal gas. From Saumon et al. (2006), we adopt $\log _{10} f_{\mathrm{H}_{2} \mathrm{O}}=-3.1, \log _{10} f_{\mathrm{CH}_{4}}=-3.3$, and $\log _{10} f_{\mathrm{NH}_{3}}=-4.9$. Note that non-equilibrium chemistry reduces $\mathrm{NH}_{3}$ abundances by a factor of 10 . 

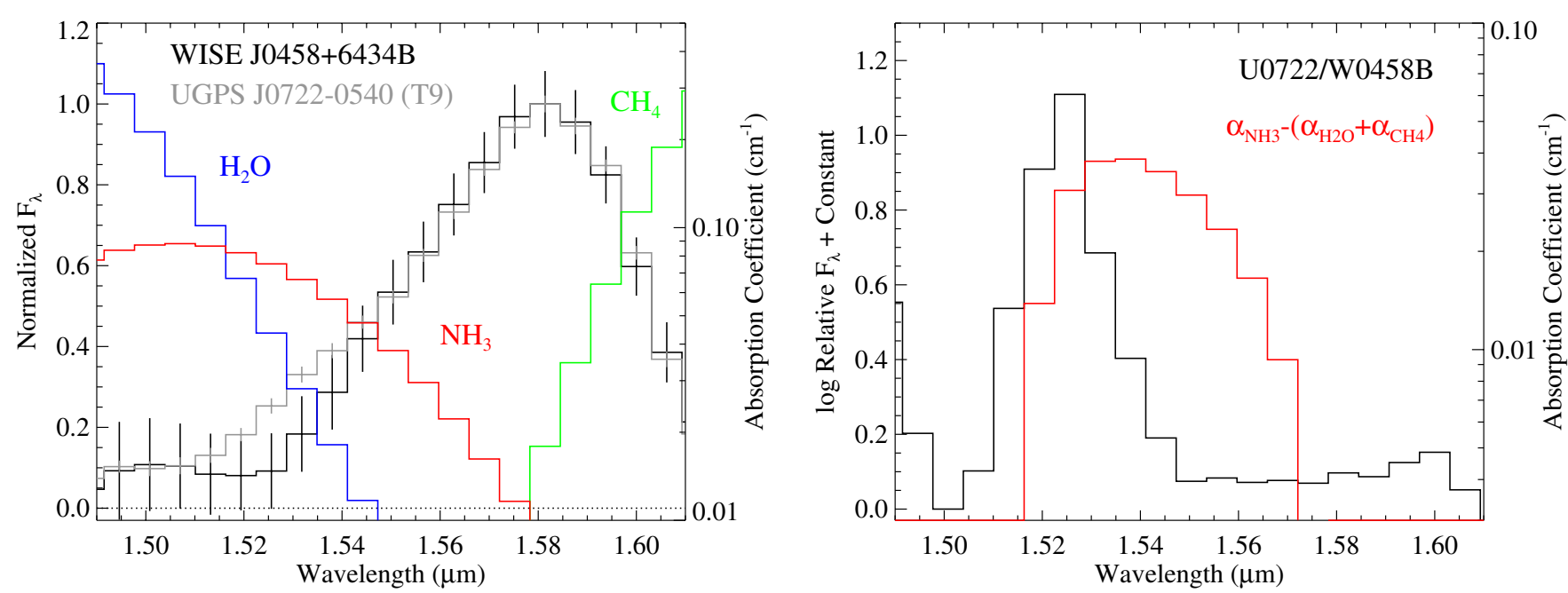

Figure 6. Left: $1.49-1.61 \mu \mathrm{m}$ spectrum of WISE J0458+6434B (black line) and UGPS J0722-0540 (gray line), both smoothed to a common resolution of $\lambda / \Delta \lambda=$ 100 (uncertainties are indicated by error bars). Overplotted are wavelength-dependent absorption coefficients for $\mathrm{H}_{2} \mathrm{O}$ (blue), $\mathrm{CH}_{4}(\mathrm{green})$, and $\mathrm{NH}_{3}$ (red), based on scattering cross-sections from Freedman et al. (2008) at $T=500 \mathrm{~K}$ and $P=1 \mathrm{bar}$, and assuming non-equilibrium abundances from Saumon et al. (2006). Note the region longward of $1.52 \mu \mathrm{m}$ where $\mathrm{NH}_{3}$ opacity is predicted to dominate over $\mathrm{H}_{2} \mathrm{O}$. Right: relative flux between UGPS J0722-0540 and WISE J0458+6434B (black line) on a logarithmic scale, compared to the relative absorption coefficient between $\mathrm{NH}_{3} \mathrm{vs} \mathrm{H}_{2} \mathrm{O}$ and $\mathrm{CH}_{4}$ combined (red line). The peak in relative flux is near the peak in relative absorption.

(A color version of this figure is available in the online journal.)

Figure 6 compares the smoothed absorption coefficients for these molecules to equivalently smoothed spectra of UGPS J0722-0540 and WISE J0458+6434B. We find that $\mathrm{NH}_{3}$ absorption exceeds that of $\mathrm{H}_{2} \mathrm{O}$ beyond $1.52 \mu \mathrm{m}$, around the region where we also see excess absorption in the spectrum of WISE J0458+6434B. The right panel of Figure 6 shows another view of this, comparing the relative absorption between UGPS J0722-0540 and WISE J0458+6434B to the differential absorption between $\mathrm{NH}_{3}, \mathrm{H}_{2} \mathrm{O}$, and $\mathrm{CH}_{4}$. There is relatively close spectral alignment between excess $\mathrm{NH}_{3}$ opacity and excess absorption in the spectrum of WISE J0458+6434B, suggesting $\mathrm{NH}_{3}$ as the primary absorber at these wavelengths. However, this conclusion carries a few caveats. First, the photospheres of brown dwarfs are not homogeneous, and absorption contributing to this spectral region is integrated over a path length that spans a range of temperatures and pressures that do not necessary coincide with the values used here to calculate molecular opacities. Second, the opacities of absorbers other than $\mathrm{NH}_{3}$ are also likely to differ between these two sources, which may add additional structure to the relative intensities. Finally, while the excess absorption in WISE J0458+6434B is statistically significant, the precise location of the relative intensity peak is not robust due to the large uncertainties in the spectrum of WISE J0458+6434B at these (low intensity) wavelengths. Hence, while evidence for $\mathrm{NH}_{3}$ absorption in the near-infrared spectrum of WISE J0458+6434B is compelling, confirmation of this will require higher signal-to-noise data and more detailed modeling of the spectrum.

\section{DISCUSSION}

One of the benefits of studying a physical binary is that its components share a common distance. In the case of WISE $\mathrm{J} 0458+6434 \mathrm{AB}$, this allows us to bootstrap existing absolute magnitude/spectral type trends out to the end of the $\mathrm{T}$ dwarf sequence. Figure 7 shows absolute $\mathrm{MKO}^{5} J$ and $H$ magnitudes

\footnotetext{
5 Mauna Kea Observatory filter system; see Tokunaga et al. (2002) and Simons \& Tokunaga (2002).
}

Table 3

Component Photometry and Distances for WISE J0458+6434AB

\begin{tabular}{lcc}
\hline \hline Parameter & WISE J0458+6434A & WISE J0458+6434B \\
\hline MKO $J$ & $17.50 \pm 0.07$ & $18.48 \pm 0.07$ \\
MKO $M_{J}$ & $17.4 \pm 0.5$ & $18.4 \pm 0.5$ \\
MKO $H$ & $17.77 \pm 0.11$ & $18.79 \pm 0.11$ \\
MKO $M_{H}$ & $17.8 \pm 0.5$ & $18.8 \pm 0.5$ \\
Est. distance (pc) & $10.5 \pm 1.8$ & $11.2 \pm 2.2$ \\
\hline
\end{tabular}

Notes. Component brightnesses are based on the new NIRC2 relative photometry reported here, combined light photometry from Mainzer et al. (2011), and the 2MASS-MKO filter corrections given in Gelino et al. (2011). Absolute magnitudes are based on the relations shown in Figure 7.

for 27 unresolved T6-Y0 dwarfs with reported parallax distance measurements. ${ }^{6}$ For sources with types later than T8, we adopt the revised classifications listed in Cushing et al. (2011). To delineate trends in absolute magnitudes from T6 to T9, we generated a series of second-order polynomial fits to these data accounting for uncertainties through Monte Carlo sampling. ${ }^{7}$ Figure 7 shows the resulting mean trends and $1 \sigma$ deviations in $M_{J}$ and $M_{H}$ as a function of spectral type, which were used to estimate the absolute magnitudes for WISE J0458+6434A and B (Table 3). Combining absolute and apparent magnitudes for each component in both filter bands, we derive statistically consistent distance estimates of $10.5 \pm 1.8 \mathrm{pc}$ and $11.2 \pm$

\footnotetext{
6 These are based on data from Tinney et al. (2003), McCaughrean et al. (2004), Vrba et al. (2004), Henry et al. (2006), Burningham et al. (2009), Artigau et al. (2010), Goldman et al. (2010), Leggett et al. (2010), Scholz (2010), Lucas et al. (2010), Marocco et al. (2010), Kirkpatrick et al. (2011), and Liu et al. (2011). We excluded two sources from this sample: the T6.5 SDSSp J134646.45-003150.4, for which existing photometry is blended with an unassociated background source (A. J. Burgasser et al., in preparation) and the T6.5p ULAS J115038.79+094942, which has a poorly constrained parallax $\left(\sigma_{\pi} / \pi=0.45\right.$; Marocco et al. 2010).

7 We assumed classification uncertainties of 0.5 subtypes and absolute magnitude uncertainties as indicated. We then performed 1000 fits to simulated data, offsetting the original spectral types and absolute magnitudes of each source by random draws from a normal distribution with mean zero and standard deviation equal to the uncertainty.
} 

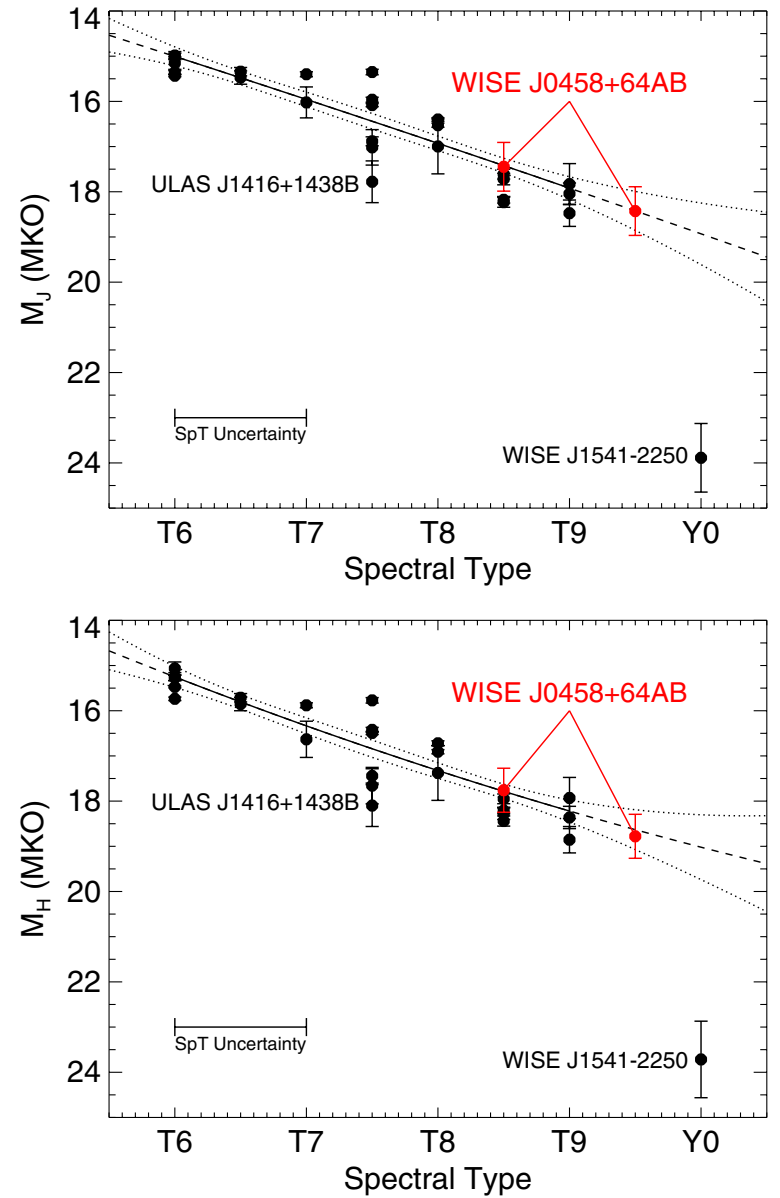

Figure 7. Absolute MKO $J$ - (top) and $H$-band (bottom) magnitudes vs. spectral type for $27 \mathrm{~T} 6-\mathrm{Y} 0$ dwarfs with measured parallaxes. Photometric uncertainties are indicated; spectral type uncertainties are assumed to be \pm 0.5 subtypes. Second-order polynomial fits to the T6-T9 dwarfs based on Monte Carlo uncertainty sampling yield a mean relation indicated by the solid line (dashed for extrapolation beyond T9) and $1 \sigma$ uncertainties indicated by the dotted lines. The estimated absolute magnitudes and uncertainties for WISE J0458+6434A based on this relation are indicated, as well as those for WISE J0458+6434B assuming that it lies at the same distance as the A component.

(A color version of this figure is available in the online journal.)

$2.2 \mathrm{pc}$ for the A and B components, respectively. These are also formally consistent with the systemic spectrophotometric estimates of Mainzer et al. (2011, 9.0 $\pm 1.9 \mathrm{pc})$ and Gelino et al. $(2011,10.5 \pm 1.4 \mathrm{pc})$. The agreement between these distances further supports the identification of this system as a physical binary or, assuming that, justifies the extrapolation of existing absolute magnitude/spectral type trends to subtype T9.5.

Currently, the only Y dwarf with a parallax distance measurement is WISEP J154151.65-225025.2 (hereafter WISE J1541-2250), classified Y0 by Cushing et al. (2011). Kirkpatrick et al. (2011) report a preliminary parallax of $351 \pm 108$ mas for this source, corresponding to a distance of $2.8 \pm 0.9 \mathrm{pc}$ and absolute magnitudes $M_{J}=23.9 \pm 0.8$ and $M_{H}=23.7 \pm 0.8$. These values make WISE J1541-2250 roughly 100 times fainter than WISE J0458+6434B in the near-infrared, despite the seemingly minor change in spectral morphology (cf. comparison of WISE J0458+6434B to the Y0 standard WISE J1738+2732 in Figure 5). This jump in brightness, also discussed by Cushing et al. (2011), suggests a dramatic redistribution of radiation out of the near-infrared band, as the corresponding difference in $T_{\text {eff }}$, roughly $500 \mathrm{~K}$ to $350 \mathrm{~K}$, reduces the luminosity by only a factor of four, assuming equivalent radii. A greater fraction of flux is certainly being emitted at mid-infrared wavelengths, due to the redward shift in the blackbody peak and increased near-infrared absorption from $\mathrm{H}_{2} \mathrm{O}$, $\mathrm{CH}_{4}$, and collision-induced $\mathrm{H}_{2}$ with lower $T_{\text {eff }}$. Additional absorption may also arise from water ice clouds that are expected to form at these temperatures, as scattering opacity from 10 to $100 \mu \mathrm{m}$ sized ice grains would efficiently suppress nearinfrared emission just as mineral grains are observed to do so in the L dwarfs (Ackerman \& Marley 2001). While an improved parallax measurement for WISE J1541-2250 and other newly discovered $\mathrm{Y}$ dwarfs are needed to confirm this dramatic drop in near-infrared flux, these early indications suggest that the $\mathrm{T} / \mathrm{Y}$ transition, like other late spectral class transitions, may trace significant changes in atmospheric chemistry as well as declining temperatures.

The authors thank Keck observing assistants Terry Stickel and Cynthia Wilburn, and instrument scientists Scott Dahm and Hien Tran, for their assistance in the observations reported here. We also acknowledge guidance on OSIRIS data reduction from Breann Sitarski and Shelley Wright, and Richard Freedman for providing scattering cross-section spectra for various molecules. We thank our referee for her/his helpful and prompt review. This work was supported by a NASA Keck PI Data Award, administered by the NASA Exoplanet Science Institute. This research has made use of the SIMBAD database, operated at CDS, Strasbourg, France; the M, L, and T dwarf compendium housed at http://dwarfarchives.org; and the SpeX Prism Spectral Libraries at http://www.browndwarfs.org/spexprism. The authors recognize and acknowledge the very significant cultural role and reverence that the summit of Mauna Kea has always had within the indigenous Hawaiian community. We are most fortunate to have the opportunity to conduct observations from this mountain.

Facility: Keck:II (NIRC2, OSIRIS, LGSAO)

\section{REFERENCES}

Ackerman, A. S., \& Marley, M. S. 2001, ApJ, 556, 872

Artigau, É., Radigan, J., Folkes, S., et al. 2010, ApJ, 718, L38

Bate, M. R. 2009, MNRAS, 392, 590

Becklin, E. E., \& Zuckerman, B. 1988, Nature, 336, 656

Bochanski, J. B., Burgasser, A. J., Simcoe, R. A., \& West, A. A. 2011, AJ, 142, 169

Burgasser, A. J. 2004, ApJS, 155, 191

Burgasser, A. J. 2007, ApJ, 659, 655

Burgasser, A. J., Geballe, T. R., Leggett, S. K., Kirkpatrick, J. D., \& Golimowski, D. A. 2006a, ApJ, 637, 1067

Burgasser, A. J., Kirkpatrick, J. D., Brown, M. E., et al. 2002a, ApJ, 564, 421 Burgasser, A. J., Kirkpatrick, J. D., Cruz, K. L., et al. 2006b, ApJS, 166, 585 Burgasser, A. J., Marley, M. S., Ackerman, A. S., et al. 2002b, ApJ, 571, L151 Burgasser, A. J., McElwain, M. W., Kirkpatrick, J. D., et al. 2004, AJ, 127, 2856 Burningham, B., Pinfield, D. J., Leggett, S. K., et al. 2009, MNRAS, 395, 1237 Burrows, A., Sudarsky, D., \& Lunine, J. I. 2003, ApJ, 596, 587

Christensen, U. R., Holzwarth, V., \& Reiners, A. 2009, Nature, 457, 167

Cooper, C. S., Sudarsky, D., Milsom, J. A., Lunine, J. I., \& Burrows, A. 2003, ApJ, 586, 1320

Cushing, M. C., Kirkpatrick, J. D., Gelino, C. R., et al. 2011, ApJ, in press (arXiv:1108.4678)

Cushing, M. C., Vacca, W. D., \& Rayner, J. T. 2004, PASP, 116, 362

Delorme, P., Delfosse, X., Albert, L., et al. 2008a, A\&A, 482, 961

Delorme, P., Willott, C. J., Forveille, T., et al. 2008b, A\&A, 484, 469

Dupuy, T. J., Liu, M. C., \& Ireland, M. J. 2009, ApJ, 692, 729

Freedman, R. S., Marley, M. S., \& Lodders, K. 2008, ApJS, 174, 504

Gelino, C. R., Kirkpatrick, J. D., Cushing, M. C., et al. 2011, AJ, 142, 57

Gelino, C. R., Marley, M. S., Holtzman, J. A., Ackerman, A. S., \& Lodders, K. 2002, ApJ, 577, 433

Gizis, J. E., Monet, D. G., Reid, I. N., et al. 2000, AJ, 120, 1085 
Goldman, B., Marsat, S., Henning, T., Clemens, C., \& Greiner, J. 2010, MNRAS, 405,1140

Griffith, C. A., \& Yelle, R. V. 1999, ApJ, 519, L85

Helling, C., Dehn, M., Woitke, P., \& Hauschildt, P. H. 2008, ApJ, 675, L105

Henry, T. J., Jao, W.-C., Subasavage, J. P., et al. 2006, AJ, 132, 2360

Kirkpatrick, J. D. 2005, ARA\&A, 43, 195

Kirkpatrick, J. D., Cushing, M. C., Gelino, C. R., et al. 2011, ApJS, in press (arXiv:1108.4677K)

Knapp, G. R., Leggett, S. K., Fan, X., et al. 2004, AJ, 127, 3553

Konopacky, Q. M., Ghez, A. M., Barman, T. S., et al. 2010, ApJ, 711, 1087

Krabbe, A., Gasaway, T., Song, I., et al. 2004, Proc. SPIE, 5492, 1403

Lane, B. F., Zapatero Osorio, M. R., Britton, M. C., Martín, E. L., \& Kulkarni, S. R. 2001, ApJ, 560, 390

Larkin, J., Barczys, M., Krabbe, A., et al. 2006, Proc. SPIE, 6269, 62691A

Lawrence, A., Warren, S. J., Almaini, O., et al. 2007, MNRAS, 379, 1599

Leggett, S. K., Burningham, B., Saumon, D., et al. 2010, ApJ, 710, 1627

Leggett, S. K., Marley, M. S., Freedman, R., et al. 2007, ApJ, 667, 537

Liu, M. C., Delorme, P., Dupuy, T. J., et al. 2011, ApJ, 740, 108

Liu, M. C., Leggett, S. K., Golimowski, D. A., et al. 2006, ApJ, 647, 1393

Lodders, K. 1999, ApJ, 519, 793

Lodders, K., \& Fegley, B., Jr. 2006, in Chemistry of Low Mass Substellar Objects (Astrophysics Update 2), ed. J. W. Mason (Heidelberg: Springer), 1

Looper, D. L., Gelino, C. R., Burgasser, A. J., \& Kirkpatrick, J. D. 2008, ApJ, 685,1183

Lucas, P. W., Tinney, C. G., Burningham, B., et al. 2010, MNRAS, 408, L56

Luhman, K. L., Burgasser, A. J., \& Bochanski, J. J. 2011, ApJ, 730, L9

Mainzer, A., Cushing, M. C., Skrutskie, M., et al. 2011, ApJ, 726, 30
Marocco, F., Smart, R. L., Jones, H. R. A., et al. 2010, A\&A, 524, A38

McCaughrean, M. J., Close, L. M., Scholz, R.-D., et al. 2004, A\&A, 413, 1029 Metchev, S. A., Kirkpatrick, J. D., Berriman, G. B., \& Looper, D. 2008, ApJ, 676,1281

Mohanty, S., Basri, G., Shu, F., Allard, F., \& Chabrier, G. 2002, ApJ, 571, 469 Monet, D. G., Levine, S. E., Canzian, B., et al. 2003, AJ, 125, 984

Nakajima, T., Oppenheimer, B. R., Kulkarni, S. R., et al. 1995, Nature, 378, 463

Noll, K. S., Geballe, T. R., \& Marley, M. S. 1997, ApJ, 489, L87

Pravdo, S. H., Shaklan, S. B., Wiktorowicz, S. J., et al. 2006, ApJ, 649, 389

Saumon, D., Marley, M. S., Cushing, M. C., et al. 2006, ApJ, 647, 552

Scholz, R. 2010, A\&A, 510, L8

Simons, D. A., \& Tokunaga, A. 2002, PASP, 114, 169

Stassun, K. G., Mathieu, R. D., \& Valenti, J. A. 2006, Nature, 440, 311

Tinney, C. G., Burgasser, A. J., \& Kirkpatrick, J. D. 2003, AJ, 126, 975

Tokunaga, A. T., Simons, D. A., \& Vacca, W. D. 2002, PASP, 114, 180

Tsuji, T. 2005, ApJ, 621, 1033

Tsuji, T., Ohnaka, K., Aoki, W., \& Nakajima, T. 1996, A\&A, 308, L29 Vacca, W. D., Cushing, M. C., \& Rayner, J. T. 2003, PASP, 115, 389

van Dam, M. A., Bouchez, A. H., Le Mignant, D., et al. 2006, PASP, 118, 310

Vrba, F. J., Henden, A. A., Luginbuhl, C. B., et al. 2004, AJ, 127, 2948

West, A. A., Hawley, S. L., Walkowicz, L. M., et al. 2004, AJ, 128, 426

Wizinowich, P. L., Le Mignant, D., Bouchez, A. H., et al. 2006, PASP, 118, 297

Wright, E. L., Eisenhardt, P. R. M., Mainzer, A. K., et al. 2010, AJ, 140, 1868

Yamamura, I., Tsuji, T., \& Tanabé, T. 2010, ApJ, 722, 682

Zapatero Osorio, M. R., Lane, B. F., Pavlenko, Y., et al. 2004, ApJ, 615, 958 